\title{
ACTIVIDAD ANTIOXIDANTE E INMUNOLÓGICA DE FLAVONOIDES AISLADOS DE HOJAS DE Smallanthus sonchifolius (YACÓN)
}

\section{Antioxidant activity and immunological of flavonoids isolated from leaves of Smallanthus sonchifolius (yacon)}

\author{
Enrique Aguilar F. ${ }^{1}$, Pablo Bonilla R. ${ }^{2}$
}

${ }^{1}$ Universidad Nacional de San Cristóbal de Huamanga. Ayacucho-Perú. ${ }^{2}$ Instituto de Investigación en Ciencias Farmacéuticas y Recursos Naturales “Juan de Dios Guevara”, Facultad de Farmacia y Bioquímica. Universidad Nacional Mayor de San Marcos. Lima-Perú.

\section{RESUMEN}

El presente trabajo de investigación se desarrolló con el objetivo de aislar y elucidar la estructura química de los flavonoides de las hojas de Smallanthus sonchifolius y determinar su actividad antioxidante e inmunológica en ratones albinos Balb/C. Las hojas fueron colectadas en el departamento de Ayacucho y sometidas a un proceso de extracción hidroalcohólica, desengrasado con éter de petróleo y extracción de los flavonoides con acetato de etilo. Los flavonoides fueron aislados por cromatografía en capa fina y sus estructuras elucidadas por espectrofotometría ultravioleta, utilizando las tablas de Mabry y col. Se determinó su bioactividad a la Artemia salina y la toxicidad aguda mediante la prueba de dosis límite. La actividad antioxidante fue evaluada utilizando la actividad secuestradora del DPPH, actividad inhibidora de la formación del radical hidroxilo y lipoperoxidación en microsomas hepáticos; y la actividad inmunológica se evaluó en ratones inmunosuprimidos experimentalmente con ciclofosfamida. Se obtuvo un rendimiento de fracción flavónica de $1 \%$ y los flavonoides aislados fueron: 5,7-dihidroxi-4'-metoxiflavonol, 5,7,3'-trihidroxi-4'-metoxiflavonol, 5-hidroxi- 4'-metoxi-7-O-glicosilflavona y 7,4'-dihidroxi-3,5'-dimetoxiflavona, los que demostraron tener actividad antioxidante semejante a la rutina, quercetina y vitamina $\mathrm{C}$, dependiente de la concentración ( $\mathrm{p}<0.05$ ); y la actividad inmunológica al estimular el aumento del número de los glóbulos blancos y rojos en animales inmunosuprimidos y sin evidencia de toxicidad significativa. Se concluye que, los flavonoides presentes en las hojas de Smallanthus sonchifolius tienen actividad antioxidante, inmunológica y no muestran toxicidad a las dosis ensayadas.

Palabras clave: Smallanthus sonchifolius, flavonoides, antioxidante, actividad inmunológica.

\begin{abstract}
This work was carried out with the purpose to isolate and structural chemistry elucidate the flavonoids of the leaf from the Smallanthus sonchifolius and to determine his antioxidant and immunological activity in mice Balb/C. The samples were collected in the department of Ayacucho and were extracted with ethanol 80\%, while that fats, waxes, and chlorophyll removed with petroleum ether, the aqueous layer was extracted with ethyl acetate. The flavonoids were isolated from the ethyl acetate extract for thin layer chromatography preparative, using the solvents system BAW (4:1:5) and visualized with ultraviolet light at $366 \mathrm{~nm}$. The flavonoids structure was elucidated by ultraviolet spectroscopic, using the Mabry et al tables. It was determined the bioassays utilizing brine shrimp (Artemia salina) and the acute oral toxicity for the doses limit test in mice. The antioxidant activity was evaluated for DPPH scavenger, inhibition the liberation de hydroxyl radical and lipid peroxidation in hepatic microsomal. The immunological activity was evaluated experimentaly in inmmunosupressed mice with cyclophosphamide. It was obtained $1 \%$ yielding of flavonic fraction. The flavonoids isolated were: 5 , 7-dihydroxy-4'methoxyflavonol, 5, 7, 3'-trihydroxy-4'-methoxyflavonol, 5-hydroxy-4'-methoxy-7-O-glycosilflavone and 7,4'-dihydroxy-3,5'dimethoxyflavone, that to demonstrate have antioxidant activity similarly to rutin, quercetin and vitamin $C$, concentration dependent $(\mathrm{p}<0.05)$ and the immunological activity, stimulating the increase the number of the both white blood and red blood cells in inmunosupressed mice and without evidence significant toxicity. It was conclude that the flavonoids in leaf of Smallanthus sonchifolius have antioxidant and immunological activity and any evidence of toxicity at.
\end{abstract}

Key words: Smallanthus sonchifolius, flavonoids, antioxidant, immunological activity.

\section{INTRODUCCIÓN}

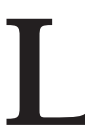

a especie originaria de los Andes sudamericanos Smallanthus sonchifolius "yacón" (Fam. Asteraceae) ${ }^{(1)}$, ha despertado renovado interés por su contenido de compuestos fenólicos en las hojas y raíces reservantes ${ }^{(2-4)}$; las hojas han demostrado tener actividad hipoglicemiante ${ }^{(5)}$, antioxidante ${ }^{(6)}$, sobre el metabolismo hepático ${ }^{(7)}$. Los flavonoides han demostrado tener una gran actividad biológica, principalmente como antioxidantes ${ }^{(8-11)}$. Se pretende 
aislar y elucidar los flavonoides de las hojas y demostrar su actividad antioxidante e inmunológica.

\section{MATERIALES Y MÉTODOS}

\section{Material biológico}

Se utilizaron 24 ratones albinos Balb/C, adquiridos en el Instituto Nacional de Salud, Chorrillos Lima, de 15-24 gramos, 12 de cada sexo para ver toxicidad y, para el estudio inmunológico 40 ratones machos de 15-24 gramos. Todos en buen estado de salud, acondicionados en jaulas del Bioterio de los Laboratorios de Farmacia de la Facultad de Ciencias Biológicas de la Universidad Nacional de San Cristóbal de Huamanga mantenidos con alimento balanceado (ratonina) y agua ad limitum, por una semana antes de los ensayos.

Las hojas de Smallanthus sonchifolius "yacón" fueron colectadas del Campo Experimental del Instituto de Investigación y Experimentación Agraria de Ayacucho (INIEA), a 2700 m.s.n.m., desecadas a temperatura ambiente y pulverizadas utilizando un molino de cuchillas, obteniéndose un polvo seco de color verde oscuro, almacenándose en frascos ámbar. Se realizó una extracción con etanol al $80 \%$ por un proceso de doble extracción, evaporación a sequedad del extracto, desengrasado con éter de petróleo (con la finalidad de eliminar grasas, ceras, pigmentos y otros metabolitos que puedan interferir con la extracción de flavonoides), seguido de ensayo de solubilidad. Luego se hizo una extracción líquido-líquido con acetato de etilo utilizando un embudo de separación, para recuperar finalmente la fracción de acetato de etilo y evaporarla a sequedad.

\section{Ensayos biológicos}

\section{Bioactividad sobre la Artemia salina $L$.}

Se utilizó la metodología descrita por Cáceres, y Meyer et al ${ }^{(12,13)}$.

\section{Toxicidad aguda}

Se utilizó el Método de Dosis Límite en ratones Balb/c de ambos sexos, según Betancourt, $2004^{(14)}$.

\section{Actividad Antioxidante}

Actividad secuestradora del radical 1,1-difenil-2picrilhidrazilo ${ }^{(15)}$.

La técnica empleando el radical DPPH (1,1-difenil-2-picril hidrazilo hidratado), que tiene un electrón desapareado y es de color azul-violeta, se basa en la desaparición de dicho color hacia el amarillo pálido por la reacción de la sustancia antioxidante, pudiendo cuantificarse la reacción espectrofotométricamente a $515 \eta \mathrm{m}$ por diferencia de absorbancias, con lo que se determina el porcentaje de inhibición del radical DPPH.

Cálculos:

$$
\begin{array}{r}
\% \text { Actividad } \\
\text { antioxidante }
\end{array}=\frac{1-\left(A_{2}-A_{3}\right)}{A_{3}} \times 100
$$

Dónde:

$A_{1}$ : Absorbancia del patrón de referencia

$\mathrm{A}_{2}$ : Absorbancia de la muestra

$\mathrm{A}_{3}$ : Absorbancia del blanco de la muestra

\section{Actividad secuestradora del radical hidroxilo ${ }^{(16)}$.}

La técnica para generar los radicales hidróxilo se basa en la reacción del hierro con el peróxido de hidrógeno, llamada Reacción de Fenton:

$$
\mathrm{Fe}^{+2}-\mathrm{EDTA}+\mathrm{H}_{2} \mathrm{O}_{2} \rightarrow \mathrm{Fe}^{+3}-\mathrm{EDTA}+\mathrm{OH}+\mathrm{OH}
$$

La lectura espectrofotométrica se realiza a $412 \mathrm{\eta m}$.

Para la estimación de la actividad captadora de radical hidroxilo de la sustancia problema, los resultados se expresan como porcentaje de inhibición de la producción de formaldehído respecto de la reacción control (ausencia del producto a ensayar).

$$
\% \text { Inhibición }=\frac{C-P}{C} \times 100
$$

Siendo:

$C=$ Reacción control

$\mathrm{P}=$ Reacción con el compuesto problema

Actividad inhibidora de la lipoperoxidación lípidica microsomal ${ }^{(15)}$.

Se emplea el modelo de la inhibición de la peroxidación producida por un prooxidante como el peróxido de hidrógeno. La inhibición se evidencia por una disminución en la producción de malondialdehído, producto de la peroxidación lípidica.

Se utilizó la fracción de acetato de etilo del extracto alcohólico de las hojas de Smallanthus sonchifolius a las concentraciones de 30, 150 y $300 \mu \mathrm{g}$, y como estándares los flavonoides rutina y quercetina, y la vitamina C, a las mismas concentraciones. 


\section{Actividad inmunológica}

Efecto de la fracción flavónica de las hojas de Smallanthus sonchifolius sobre la inmunosupresión inducida por la ciclofosfamida ${ }^{(18)}$.

El método usado fue descrito por Ziauddin et al. Se utilizaron ratones albinos machos, de 18 a $25 \mathrm{~g}$ de peso, divididos en 5 grupos, cada uno con 8 ratones. El Grupo I, utilizado como control, recibió el vehículo carboximetilcelulosa (CMC) al o,1\% y el Grupo II de la ciclofosfamida también recibió $\mathrm{CMC}$ o,1\%, durante 15 días respectivamente. Los grupos III, IV y V recibieron la fracción de acetato de etilo a las dosis de 100, 200 y $400 \mathrm{mg} / \mathrm{kg}$ por vía oral, disuelto en $\mathrm{CMC}$ o, $1 \%$, respectivamente, durante 15 días previo a la administración de ciclofosfamida. Los grupos II al V recibieron ciclofosfamida a la dosis de 30 $\mathrm{mg} / \mathrm{kg}$, vía oral, en los siguientes tres días. En el día 19, se extrajo la sangre, por punción cardiaca, de cada uno de los animales; esta se recibió en viales estériles con anticoagulante heparina para realizar el recuento de glóbulos blancos y rojos.

\section{Análisis de datos}

Los resultados se expresan en forma de medias y su desviación estándar. La CL50 y la DL50 se determinaron utilizando el paquete estadístico Probits.

Para la significancia estadística se utilizó el Análisis de Varianza y la Prueba de Tukey al 95\% de confianza.
Tabla 1. Actividad inhibidora de la lipoperoxidación lípidica microsomal.

\begin{tabular}{lccc}
\hline \multicolumn{1}{c}{ Tubo } & Blanco & Control & Muestra \\
\hline Microsomas hepáticos (2mg/mL) & 0.5 & 0.5 & 0.5 \\
Buffer fosfato $100 \mathrm{mM} \mathrm{pH} \mathrm{7.4}$ & 1.5 & 1.4 & 1.3 \\
$\mathrm{H}_{2} \mathrm{O}_{2}$ & - & 0.1 & 0.1 \\
$\begin{array}{l}\text { Extracto o Estándar (flavonoides: } \\
\text { rutina y querecetina y vitamina C) }\end{array}$ & - & - & 0.1 \\
\hline
\end{tabular}

Leer en espectrofotómetro a $535 \mathrm{~nm}$, a esta longitud se mide el complejo $\mathrm{Ma}$ londialdehído - Acido tiobarbitúrico.

Tabla 2. Extracto etanólico al $80 \%$ de hojas de Smallanthus sonchifolius (yacón), marcha de solubilidad.

\begin{tabular}{ccc}
\hline $\begin{array}{c}\text { Muestra } \\
\text { problema } \\
\text { (mg de extracto) }\end{array}$ & $\begin{array}{c}\text { Solvente } \\
1 \mathrm{~mL}\end{array}$ & Resultados \\
\hline 5 & Éter de petróleo & + \\
5 & n-hexano & + \\
5 & Cloroformo & ++ \\
5 & Acetato de etilo & ++ \\
5 & n-butanol & ++ \\
5 & Etanol & +++ \\
5 & Metanol & +++ \\
5 & Agua & +++ \\
\hline
\end{tabular}

$(+++)$ Soluble, $\quad(++)$ Medianamente soluble, $\quad(+)$ Poco soluble

\section{RESULTADOS}

Tabla 3. Extracto etanólico al 80\% de hojas de Smallanthus sonchifolius (yacón), marcha fitoquímica.

\begin{tabular}{ccc}
\hline Reactivo & Resultado & Metabolito \\
\hline Ninhidrina & ++ & Aminoácidos libres \\
Gelatina & ++ & Taninos \\
$\mathrm{FeCl}_{3}$ & ++ & Compuestos fenólicos \\
Shinoda $\left(\mathrm{Mg}^{0}+\mathrm{HCl}\right)$ & ++ & Flavonoides \\
Dragendörff & -- & Alcaloides \\
Mayer & -- & Alcaloides \\
Hager & -- & Alcaloides \\
Wagner & -- & Alcaloides \\
Liebermann & ++ & Triterpenoides y esteroides \\
Baljet & ++ & Lactonas y cumarinas \\
Kedde & ++ & Lactonas \\
$\alpha$-naftol $+\mathrm{H}_{2} \mathrm{SO}_{4}$ & ++ & Glucósidos
\end{tabular}

(+++) Abundante; (++) bastante; (+) poco; (--)nada 
Tabla 4. Extracto etanólico al $80 \%$ de hojas de Smallanthus sonchifolius (yacón), fraccionamiento.

\begin{tabular}{cccccc}
\hline Fracción & Color & Peso & \% & Shinoda & FeCl $_{\mathbf{3}}$ \\
\hline Etérea & Verde Negruzco & $42.30 \mathrm{~g}$ & 38.77 & Negativo & Negativo \\
Acetato de Etilo & Amarillo & $10.80 \mathrm{~g}$ & 9.9 & Color Rojo Intenso & Azul Negruzco \\
Acuosa & Amarillo Rojizo & $56.00 \mathrm{~g}$ & 51.33 & Ligera coloración roja & Ligera coloración azul \\
\hline
\end{tabular}

Tabla 5. Fracción en acetato de etilo del extracto etanólico al 80\% de hojas de Smallanthus sonchifolius (yacón), cromatograma en capa fina.

\begin{tabular}{|c|c|c|c|c|c|c|}
\hline $\begin{array}{c}\text { Comp. } \\
\text { № }\end{array}$ & $\mathbf{R f}$ & Visible & $\begin{array}{c}\text { Luz Uv }{ }_{366} \\
\text { (fluorescencia) }\end{array}$ & $\mathrm{FeCl}_{3}$ & $\lambda \mathrm{UV}$ & Metabolito \\
\hline 1 & 0.875 & Marrón & Roja & Marrón Azulado & --- & Clorofila \\
\hline 2 & 0.8 & Marrón & Celeste & Azul & 258,316 & Flavonol \\
\hline 3 & 0.725 & Marrón oscuro & Púrpura intenso & Azul Intenso & $258,269,346$ & Flavonol \\
\hline 4 & 0.7 & Marrón oscuro & Púrpura intenso & Azul Intenso & $258,269,346$ & Flavonol \\
\hline 5 & 0.625 & Marrón & Púrpura & Azul & 258,322 & Flavona \\
\hline 6 & 0.5 & Marrón & Púrpura & Azul Intenso & $258,282,340$ & Flavona \\
\hline 7 & 0.425 & Marrón & Púrpura & Azul & 258 & Fenilpropanoide \\
\hline
\end{tabular}

\section{Actividad Antioxidante}

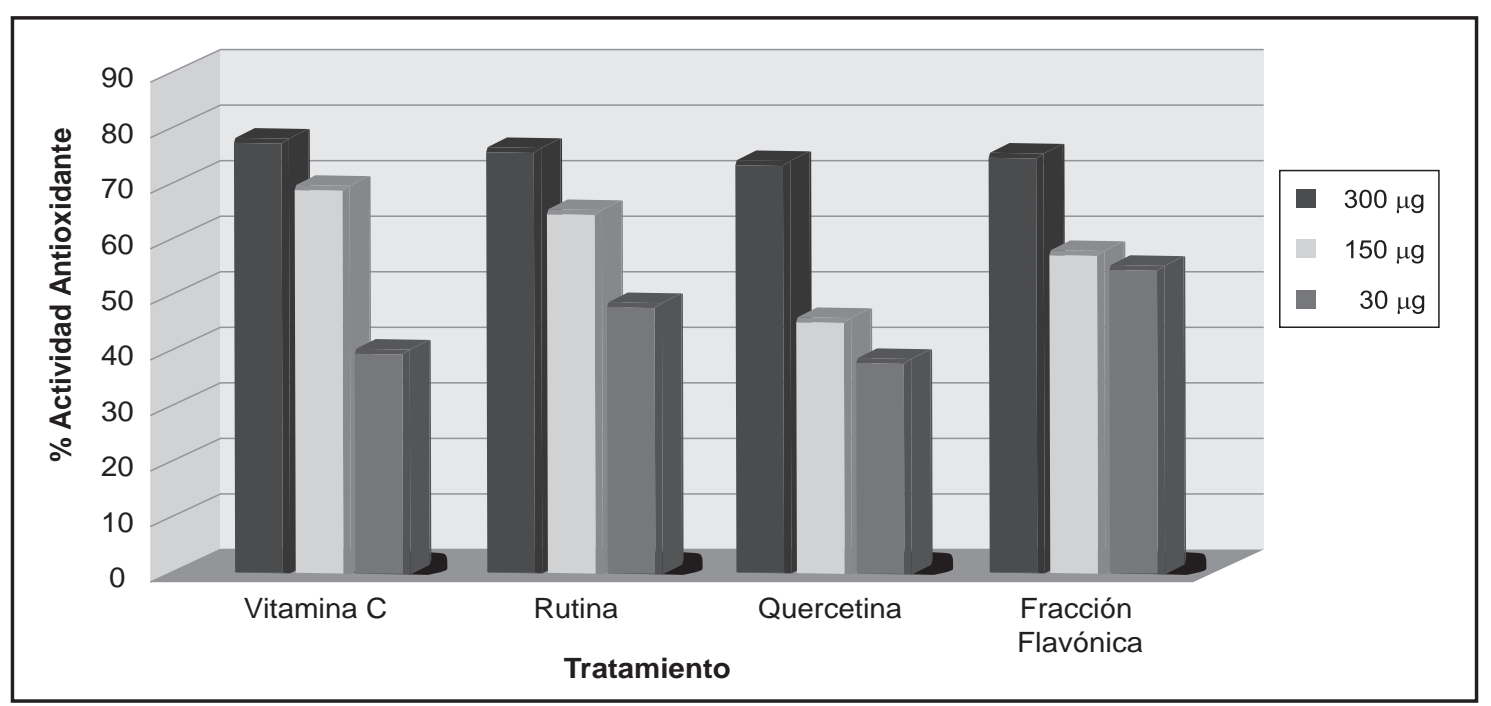

Figura 1. Fracción flavónica de las hojas de Smallanthus sonchifolius (yacón), actividad secuestradora del radical DPPH.

\section{DISCUSIÓN}

De la maceración hidroalcohólica de un kg de hojas secas, se obtuvo un rendimiento de extracto de 109,10 g, que representa un 10\%. El extracto etanólico de las hojas de Smallanthus sonchifolius "yacón" mostró tener una buena solubilidad en solventes polares como el etanol o metanol, mediana solubilidad en solventes como cloroformo y acetato de etilo; baja solubilidad en solventes poco polares como éter de petróleo y n-hexano, y en sol- ventes altamente polares como el agua; esto indica presencia de sustancias de mediana y alta polaridad (Tabla 2). La marcha fitoquímica del extracto etanólico reporta presencia de aminoácidos libres, taninos, compuestos fenólicos, flavonoides, terpenoides, azúcares, lactosas, cumarinas, y compuestos con grupo carbonilo (Tabla 3). Mediante ensayos cromatográficos se confirmó la presencia de compuestos fenólicos, flavonoides, terpenos y lactonas, utilizando como adsorbente silicagel $60-G$ y como 


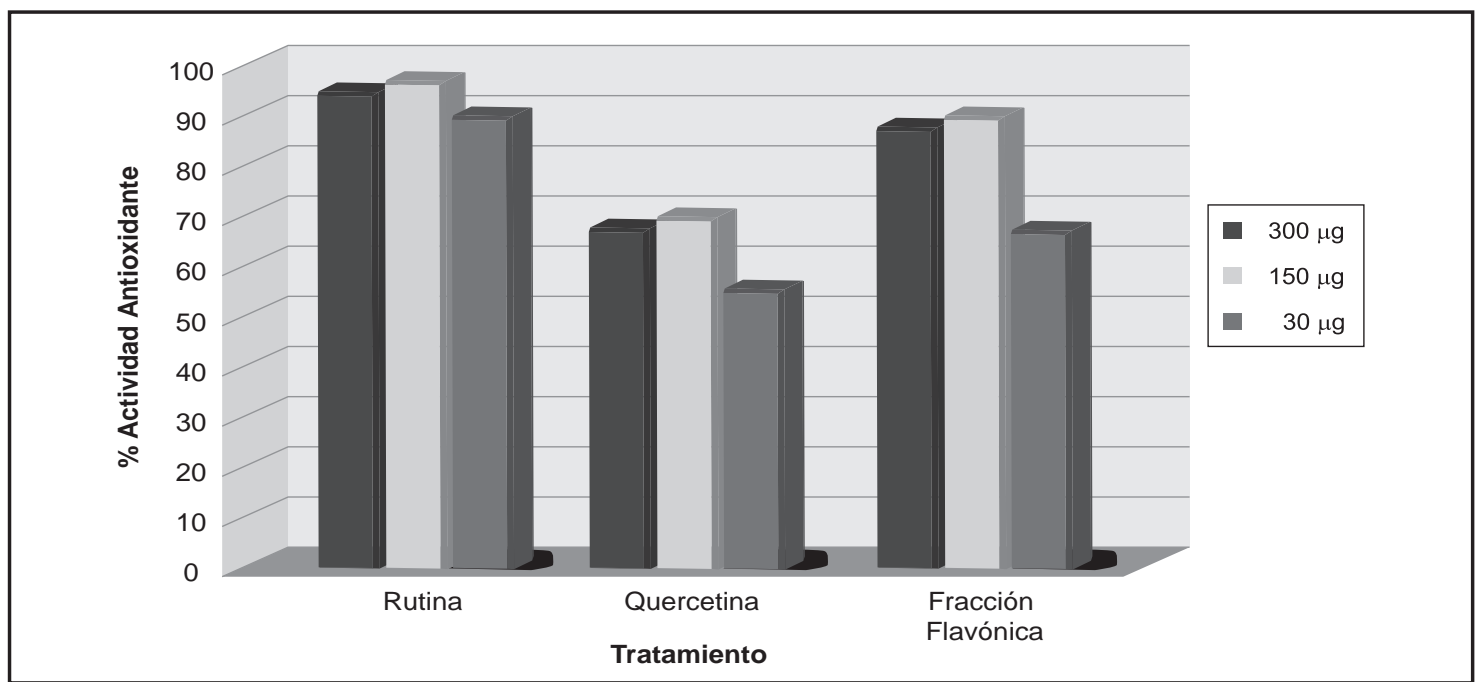

Figura 2. Fracción flavónica de las hojas de Smallanthus sonchifolius (yacón), actividad inhibidora de la formación del radical hidroxilo.

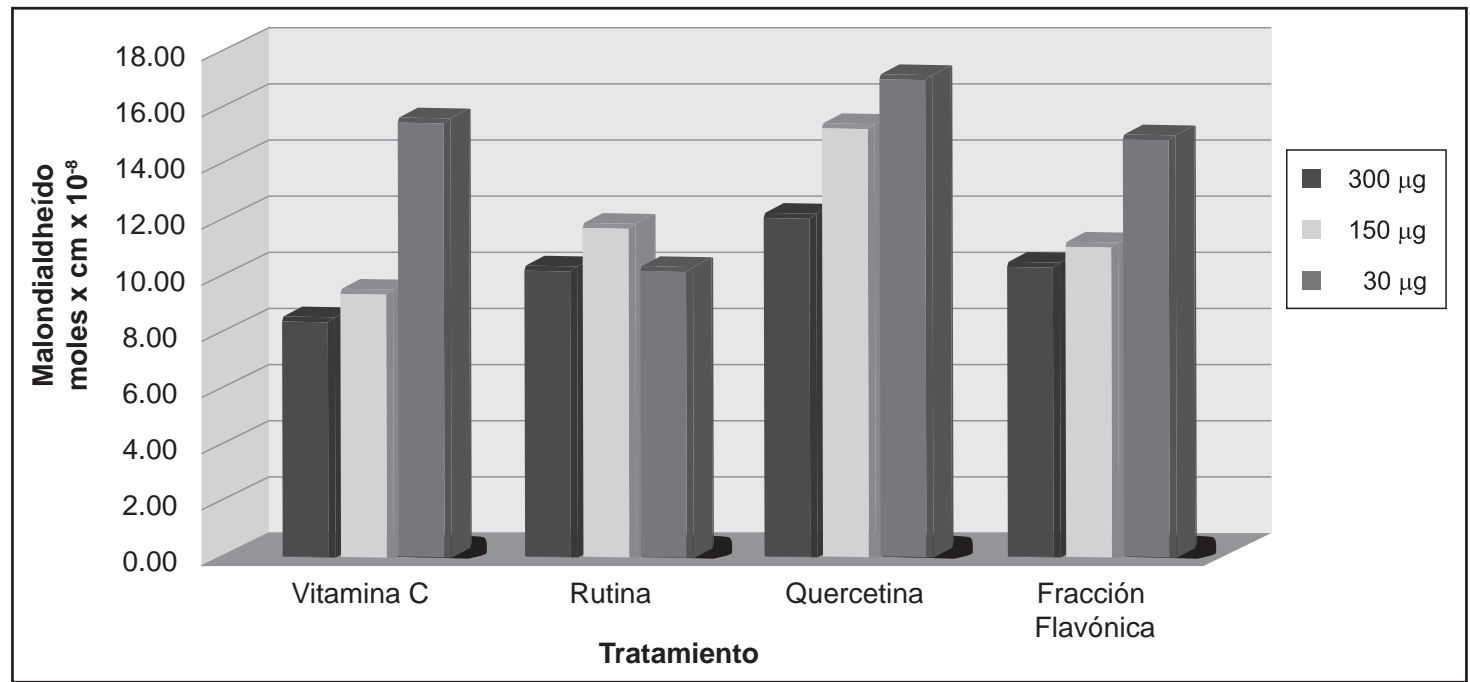

Figura 3. Fracción flavónica de las hojas de Smallanthus sonchifolius (yacón), actividad inhibidora de la formación del Malondialdehido.

fase móvil, $\mathrm{CHCl}_{3}: \mathrm{MeOH}$ (12:1); observados los cromatogramas a la lámpara de luz UV 366 ๆm se detectó presencia de flavonoides, se reveló con $\mathrm{FeCl}_{3}$ al $5 \%$ que detectó compuestos fenólicos, con el reactivo de Lieberman dio terpenos y con el de Kedde lactonas sesquiterpénicas.

Para aislar los flavonoides se procedió a realizar una extracción líquido-líquido en el extracto etanólico, primero con éter de petróleo para eliminar las grasas, después con acetato de etilo para extraer los flavonoides, quedando como residuo la fase acuosa. Se verificó la presencia de flavonoides con la reacción de Shinoda y compuestos fenólicos con $\mathrm{FeCl}_{3} 5 \%$, resultando positivo para la fracción de acetato de etilo, y ligeramente en la fracción acuosa, con un rendimiento de 9,9\% en la fracción de acetato de etilo, que representa un $1 \%$ de contenido flavónico (Tabla 4).

$\mathrm{Al}$ respecto, Valentová et al. refieren un proceso de extracción utilizando Soxhlet y metanol como solvente. En dicho procedimiento, después de eliminar el metanol, el extracto fué resuspendido en agua y desengrasado con éter de petróleo; para luego ser extraído con acetato de etilo obteniendo un rendimiento de 0,26 g, que representa el 1,3\% del total, valor ligeramente superior al nuestro. Posiblemente el mayor rendimiento se deba a que después del proceso de desengrasado, la fase acuosa fue acidificada con $\mathrm{H}_{3} \mathrm{PO}_{4} \mathrm{O}, 01 \mathrm{~mol} / \mathrm{L}$, 


\section{Actividad Inmunológica}

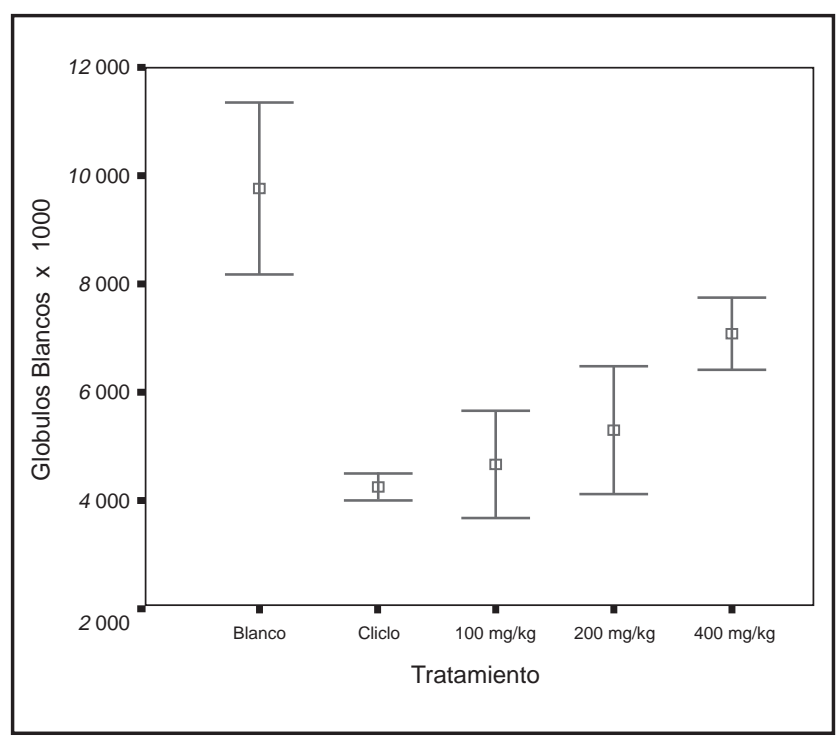

Figura 4. Fracción flavónica de las hojas de Smallanthus sonchifolius (yacón), actividad inmunológica según el número de glóbulos blancos.

extrayendo compuestos como el ácido clorogénico, ácido cafeico y ácidos dicafeilquínicos.

Los flavonoides fueron aislados y purificados mediante cromatografía en capa fina a escala preparativa, utilizándose como adsorbente silicagel 6o-G, como sistema de solventes butanol:ácido acético:agua (BAW) (4:1:5), y observados a la lámpara de luz UV 366 ๆm. Fueron recuperados del silicagel con metanol, lográndose aislar 7 fracciones, de las cuales 6 correspondían a compuestos fenólicos con probable estructura flavónica y la otra a la clorofila, por la fluorescencia roja característica. El uso del sistema BAW (4:1:5), está reportado por Lock de Ugaz ${ }^{10}$.

Las estructuras químicas de las seis fracciones aisladas fueron elucidadas mediante correlaciones químicas con los datos obtenidos por espectroscopía UV-Visible en metanol y por comparación con espectros dados en la literatura por Mabry et al. ${ }^{17}$ sugiriéndose la estructura de 4 flavonoides y un fenilpropanoide (Tabla 5).

\section{Elucidación de estructuras}

Fracción $\mathbf{N}^{\circ}$ 2. $\lambda_{\max }^{\mathrm{MeOH}}=258316 \eta \mathrm{m}$. UV 366 ๆm = fluorescencia celeste intensa, comparando con las tablas de Mabry et al. ${ }^{(17)}$ se propone la siguiente estructura: 5,7-dihidroxi-4'-metoxiflavonol.

Fracción $\mathbf{N}^{\circ} 3$ y 4. $\lambda_{\max }^{\mathrm{MeOH}}=258269346$ ๆm. UV 366

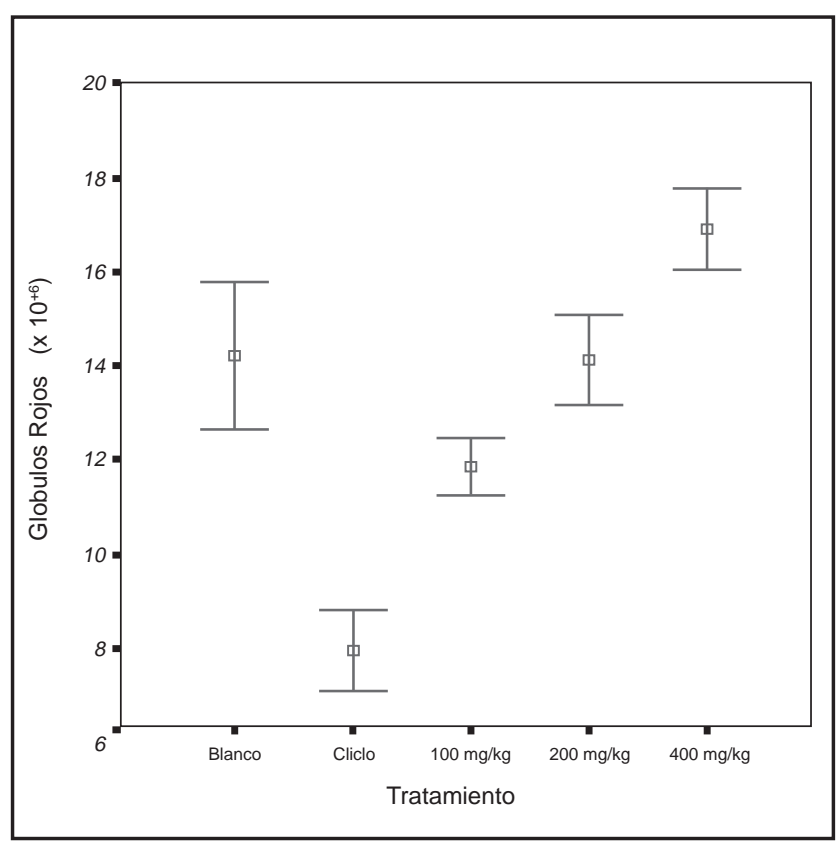

Figura 5. Fracción flavónica de las hojas de Smallanthus sonchifolius (yacón), actividad inmunológica expresada según el número de glóbulos rojos producidos.

$\eta \mathrm{m}$ = fluorescencia púrpura intensa, comparando con las tablas de Mabry et al. ${ }^{(17)}$ se propone la siguiente estructura: 5,7,3'-trihidroxi-4'-metoxiflavonol.

Fracción $N^{\circ}$ 5. $\lambda_{\max }^{\mathrm{MeOH}}=258322 \eta \mathrm{m}$. UV $366 \eta \mathrm{m}=$ fluorescencia púrpura, correlacionando los datos espectroscópicos con las tablas de Mabry et al. ${ }^{(17)}$ se propone la estructura: 5-hidroxi-4'-metoxi-7-O-glicosilflavona.

Fracción $N^{\circ} 6 . \quad \lambda_{\max }^{\mathrm{MeOH}}=258282340 \eta \mathrm{m}$. UV 366 ๆm $=$ fluorescencia púrpura, interpolando los datos con las tablas de Mabry et al. ${ }^{(17)}$ se sugiere la estructura: 7,4 'dihidroxi-3,5'dimetoxiflavona.

Fracción $\mathbf{N}^{\circ}$ 7. $\lambda_{\text {max }}^{\mathrm{MeOH}}=258 \eta \mathrm{m}$. UV $366 \eta \mathrm{m}=$ Fluorescencia púrpura, comparando con las tablas de Mabry et al. ${ }^{(17)}$ se propone la estructura de un fenilpropanoide.

Simonovska et al. (2) después de hidrolizar una fracción de acetato de etilo, de los extractos metanólicos de la raíz tuberosa y hojas, detectaron la presencia de quercetina en una cromatografía en capa fina, siendo revelado con el reactivo éster difenilborico del ácido 2-aminoetilo al 1\% en metanol (NST) lo que produjo una fluorescencia de color amarillo a la luz UV de 366 ๆm y otros dos flavonoides desconocidos; resultados que son muy cercanos al nuestro, porque el flavonoide de la fracción № 3, que se encuentra en mayor cantidad por las manchas muy intensas del cromato- 
grama, muestra ser un derivado de la quercetina. Los otros flavonoides, que los mismos autores los reportan como desconocidos, en este trabajo se les asigna la estructura correspondiente a un flavonol y una flavona. En una especie relacionada, el Smallanthus fruticosus, se ha determinado la presencia de una flavona, la centaureidina $^{(6)}$.

\section{Actividad antioxidante}

La actividad antioxidante se determinó mediante la captación del 1,1-difenil-2-picrilhidrazilo (DPPH), midiendo la actividad inhibidora de la formación de radicales libres y la producción de malondialdehído como indicador de lipoperoxidación (Figuras 1-3).

En todos los casos, la fracción flavónica mostró actividad antioxidante dependiente de la concentración, a mayor concentración mayor actividad antioxidante; pero no mostraron diferencias cuando se compara con la vitamina $\mathrm{C}$, los flavonoides estándar quercetina y rutina, tal como se muestra en sus respectivas pruebas estadísticas, donde existe significancia en las concentraciones ensayadas, pero no con las sustancias de comparación; es decir, tiene una actividad antioxidante comparativamente similar a la vitamina $C$, quercetina y rutina. Respecto a la actividad antioxidante de los flavonoides se sabe que las flavonas y sobre todo los flavonoles se muestran como los más activos contra los radicales libres ${ }^{(9)}$. El 5,7-dihidroxi-4'-metoxiflavonol y el 5,7,3'-trihidroxi-4'-metoxiflavonol que se encuentran en mayor cantidad son flavonoles, mientras que los otros flavonoides son derivados de flavonas; esto explica la actividad antioxidante de la fracción flavónica del extracto alcohólico, comparado a la rutina y quercetina que son flavonoles ${ }^{(6)}$. Utilizando la fracción de acetato de etilo, de un extracto metanólico, determinaron que el $\mathrm{IC}_{50}(\mu \mathrm{g} / \mathrm{mL})$ necesario para neutralizar al DPPH es $24.3 \pm 2.7 \mu \mathrm{g} / \mathrm{mL}$, mientras que en el presente trabajo con la fracción flavónica sin hidrolizar, el $\mathrm{IC}_{50}$ fue $24,7 \mu \mathrm{g} / \mathrm{mL}$. Los mismos autores en su prueba de inhibición de la lipoperoxidación reportan un $\mathrm{IC}_{50}$ de $32.8 \pm 2.3 \mu \mathrm{g} / \mathrm{mL}$, en nuestro caso fue de $14,10 \mu \mathrm{g} / \mathrm{mL}$, que significa que se logró una mejor protección contra el daño sobre los lípidos del microsoma hepático.

Cuando los flavonoides tienen orto sustituciones en el anillo A o B, son estructuras que tienen una buena acción inhibidora de la formación del radical hidroxilo. En nuestro caso, los flavonoides aislados de las fracciones 2 y 3 , tienen hidroxilos en posición orto en el anillo A, lo que explicaría su acción sobre dicho radical. Hirano et al. ${ }^{(19)}$ evaluaron la actividad de 10 flavonoides sobre el DPPH, demostrando que esta actividad dependía de la naturaleza química. La quercetina mostró una mayor actividad secuestradora que la luteolina.

En la Figura 3 se muestra la actividad antioxidante como inhibición de la producción de malondialdehído por lipoperoxidación de las membranas, no encontrando diferencias estadísticamente significativas entre la vitamina $C$, rutina, quercetina y la fracción flavónica de las hojas de Smallanthus sonchifolius. Posiblemente estas sustancias tienen la capacidad de actuar protegiendo las membranas de los microsomas hepáticos, penetrando los espacios acuosos y lipídicos de dichas membranas, neutralizando el radical peróxido producido por el $\mathrm{H}_{2} \mathrm{O}_{2}$. Nakagawa et al. ${ }^{(20)}$ estudiaron el efecto sobre la lipoperoxidación lipídica de la quercetina y de la rutina sobre fracciones de lisosomas hepáticos, atacados por generadores de radicales libres hidrofílicos y lipofílicos. Hallaron que la quercetina tenía un mejor efecto protector sobre las membranas biológicas que la rutina, su correspondiente glicósido, concluyendo que la quercetina tenía una gran actividad antioxidante, debido a su habilidad para penetrar las membranas de los lisosomas hepáticos.

\section{Actividad inmunológica}

El sistema inmune es altamente complejo, intrínsecamente regulado por un grupo de células cuya función integrada es esencial para la salud. La respuesta inmune podría producirse por interacciones entre las células que la conforman o mediante respuestas de los mensajeros intracelulares que incluyen a las hormonas, citoquinas y autacoides elaboradas por varias células. Los autacoides usualmente incluyen histamina, kininas, leucotrienos, prostaglandinas y serotonina. El sistema inmune puede ser modificado por la dieta, agentes farmacológicos, contaminantes ambientales, y sustancias químicas naturales como las vitaminas y los flavonoides. Los flavonoides pueden tener efectos sobre las funciones de las células T, células B, macrófagos, células NK, basófilos, células del mastocito, neutrófilos, eosinófilos y plaquetas. La actividad inmunológica de la fracción flavónica de las hojas de Smallanthus sonchifolius se determinó evaluando la respuesta inmune frente a la inmunosupresión producida por ciclofosfamida a la dosis de $30 \mathrm{mg} / \mathrm{kg}$, después de la administración por 15 días de los extractos a las dosis de 100,200 y $400 \mathrm{mg} / \mathrm{kg}$ de peso, respectivamente. La administración previa de los extractos es con la finalidad de estimular el sistema inmunológico 
y preparar al organismo para hacer frente a la ciclofosfamida, un agente inmunosupresor que produce una disminución del número de glóbulos blancos y glóbulos rojos, elementos celulares comprometidos con la respuesta inmune; asimismo, también produce una disminución de la hemoglobina como parte del daño a los eritrocitos y por ende una menor oxigenación de las células. Los glóbulos blancos son fuertemente afectados por la ciclofosfamida como se observó en el grupo tratado con dicho agente inmunosupresor, mientras que las dosis ensayadas permiten una recuperación del número de leucocitos, pero no al nivel del grupo control. La prueba estadística indica una alta significancia ( $\mathrm{p}<0.05)$, demostrando el efecto de la dosis de la fracción flavónica para atenuar el daño producido por la ciclofosfamida, estando la dosis de $400 \mathrm{mg} / \mathrm{kg}$ más cercana al grupo control que no recibió el agente inmunosupresor (Figura 4). Los glóbulos rojos también son afectados por la ciclofosfamida, tal como se muestra en el grupo tratado con el agente inmunosupresor, pero a diferencia de los glóbulos blancos, con las dosis ensayadas se logra una mejor recuperación en su número, inclusive ligeramente superior al grupo control. El análisis estadístico nos muestra una alta significancia ( $\mathrm{p}<0.05)$, indicando también el efecto de la dosis de la fracción flavónica, para neutralizar el efecto inmunosupresor de la ciclofosfamida, alcanzándose con la dosis de $200 \mathrm{mg} / \mathrm{kg}$ un número de eritrocitos semejante al grupo control y con la dosis de $400 \mathrm{mg} / \mathrm{kg}$ un número ligeramente superior al grupo no tratado. La actividad protectora sobre los glóbulos rojos se puede explicar por la capacidad de inhibir la lipoperoxidación de sus membranas por los flavonoides, (Figura 5) ${ }^{(21)}$. Estos resultados sugieren que, la fracción flavónica del extracto hidroalcohólico de las hojas de Smallanthus sonchifolius "yacón" contienen flavonoides que atenúan el efecto inmunosupresor de la ciclofosfamida y estimulan la recuperación del mismo.

Hoy se conoce que existe relación entre la actividad antiinflamatoria de los flavonoides y su actividad sobre el sistema inmune. Varios flavonoides específicamente afectan la función de sistemas enzimáticos críticamente comprometidos en la generación de los procesos inflamatorios especialmente la tirosina y la kinasa de la proteína serina-treonina. Recientemente, ha llegado a ser evidente que esas enzimas están íntimamente involucradas en la traducción de las señales del sistema inmune, así como de otras células activadas por hormonas, autacoides, neurotransmisores, y factores del crecimiento. Middleton et al. ${ }^{(22)}$ en una revisión amplia sobre los efectos de los flavonoides en las células, señalan que estos actúan modulando la respuesta de linfocitos $\mathrm{T}$, linfocitos $\mathrm{B}$, macrófagos y monocitos, mastocitos, basófilos, neutrofilos, eosinofilos y plaquetas. El aspecto estructural también es importante en la respuesta inmunológica. Por ejemplo, para inhibir la liberación de histamina, un mediador implicado en procesos alérgicos, los requerimientos estructurales son semejantes a los descritos para la actividad antioxidante anteriormente mencionados. Los flavonoles quercetina, kamferol y la miricetina son reportados como potentes inhibidores de la liberación de histamina en los mastocitos de peritoneo de ratas.

Se concluye que, los flavonoides presentes en las hojas de Smallanthus sonchifolius tienen actividad antioxidante, inmunológica y no muestran toxicidad a las dosis ensayadas.

\section{REFERENCIAS BIBLIOGRÁFICAS}

1. Grau A, Kortsarz A, Aybar M, Sánchez Riera A, Sánchez S. El Retorno del Yacón. Ciencia Hoy 2001 Junio-Julio; 11( 63).

2. Simonovska B, Vovk I, Andrenšek S, Valentová K, Ulrichová J. Investigation of phenolic acids in yacon (Smallanthus sonchifolius) leaves and tubers. Journal of Chromatography 2003; 1016:89-98.

3. Takenaka M, Yan X, Ono H, Yoshida M, Nagata T, Nakanishi T. Caffeic acid Derivatives in the Roots of Yacon (Smallanthus sonchifolius). J. Agric. Food Chem 2003; 51:793-796.

4. Takenaka M, Ono H. Novel octulosonic acid derivatives in the composite Smallanthus sonchifolius. Tetrahedron letters 2003; 44:999-1002.

5. Ayvar M, Sánchez Riera A, Grau A, Sánchez S. Hypoglycemic effect of the water extract of Smallanthus sonchifolius (yacon) leaves in normal and diabetic rats. Journal of Ethnopharmacology 2001; 74(2):125- 132.

6. Valentová K, Ulrichová J. Smallanthus sonchifolius and Lepidium meyenii-prospective Andean crops for the prevention of chronic diseases. Biomed. Papers 2003; 147(2):119-130.

7. Valentová K, Moncion A, De Waziers I, Ulrichová J. The effect of Smallanthus sonchifolius leaf extracts on rat hepatic metabolism. Cell Biology and Toxicology 2004; 20:109-120.

8. Bruneton J. Plantas medicinales. Fitoquímica y Farmacognosia. Segunda Edición 2001. Editorial Acribia. Zaragoza.

9. Villar del Fresno M. Farmacognosia general. Segunda Edición 1999. Editorial Síntesis. Madrid.

10. Lock de Ugaz O. Investigación fitoquímica: Métodos en el estudio de los productos naturales. 1994 Fondo Editorial de la Universidad Católica del Perú. Lima.

11. Kuklinski C. Farmacognosia. Estudio de las drogas y 
sustancias medicamentosas. 20oo Ediciones Omega. Barcelona.

12. Cáceres A. Plantas de uso medicinal en Guatemala. 1996 Editorial Universitaria. Universidad San Carlos de Guatemala. Guatemala.

13. Meyer B, Ferrigni N, Putnam J, Jacobsen L, Nichols D, McLaughlin J. Brine Shrimp: A convenient general bioassay for active plant constituents. Planta Medica 1982; 45:31-34.

14. Betancourt J. Resúmenes del Curso Internacional “Toxicología Experimental". Facultad de Farmacia y Bioquímica. Universidad Nacional Mayor de San Marcos. Lima. 2004.

15. Suárez S. Curso de Bioquímica Vegetal. Maestría en Recursos Vegetales y Terapéuticos. Universidad Nacional Mayor de San Marcos. Lima 2003.

16. CYTED. Manual de técnicas de investigación. Programa Iberoamericano de Ciencia y Tecnología para el Desarrollo 1995.

17. Mabry T, Markham K, Thomas M. The systematic identification of flavonoids. Springer-Verlag. New York. Heidelberg Berlin. 1970.

18. Gokhale A, Damre A, Saraf M. Investigations into the inmunomodulatory activity of Argyreia speciosa. Journal of Ethnopharmacology 2003; 84:109-114.

19. Hirano R, Sasamoto W, Matsumoto A, Itakura H, Igarashi $\mathrm{O}$ y Kondo K. Antioxidant ability of various flavonoids against DPPH radicals and LDL oxidation. J Nutr Sci Vitaminol 2001; 47(5):357-62.
20. Nakagawa K, Kawagoe M, Yoshimura M, Arata H, Minamikawa T, Nakamura M, Matsumoto A. Differential effects of flavonoid quercetin on oxidative damages induced by hydrophilic and lipophilic radical generators in hepatic lysosomal fractions of mice. J of Health Science 2000; 46(6):509-512.

21. Maridonneau-Parini I, Braquet $\mathrm{P}$, Garay RP. Heterogeneous effect of flavonoids on $\mathrm{K}+$ loss and lipid peroxidation induced by oxygen-free radicals in human red cells. Pharmacol Res Commun 1986; 18(1):61-72.

22. Middleton E, Kandaswami $C$, Theoharides T. The effects of plant flavonoids on mammalian cells: Implications for inflammation, heart disease, and cancer. Pharmacol Rev 2000; 52:673-751.

Manuscrito recibido el: 30/11/2009

Aceptado para su publicación el: 07/01/2010

\section{Correspondencia:}

Dr. Pablo Enrique Bonilla Rivera

Dirección: Jr. José María Plaza № 274, Lima 11 - Perú. E-mail: pbonillar@unmsm.edu.pe 